\section{Mortalidade infantil e serviços de Atenção Primária à Saúde em Porto Alegre (RS), Brasil}

\author{
Infant mortality and Primary Health Care Services in Porto Alegre \\ (RS), Brazil
}

\section{Mortalidad infantil y servicios de Atención Primaria a la Salud en Porto Alegre (RS), Brasil}

\section{Resumo}

Introdução: O Coeficiente de Mortalidade Infantil, indicador das condições sociais e de saúde da população, tem capacidade para orientar a proposição de políticas públicas, instalação e previsão de recursos humanos em saúde. Territórios com elevada mortalidade infantil tendem a ser mais vulneráveis e a necessitarem de uma atenção primária bem estruturada. Objetivo: Mapear a mortalidade infantil e descrever a distribuição geográfica dos serviços e recursos humanos de atenção primária (concentração e "vazios" assistenciais) em Porto Alegre, nos anos 2010 e 2014. Métodos: Estudo descritivo, delineamento ecológico, com emprego de dados secundários disponíveis em sistemas de informações em saúde. O tratamento dos dados ocorreu pelo framework Enyalius. Resultados: Dos 143 serviços, $65 \%$ possuem saúde da família, com cobertura de 36,5\% da população. Os usuários adscritos por serviço variam de 700 a 115.673 habitantes; $35 \%$ dos serviços possuem menos de 4.000 habitantes adscritos. A distribuição de médicos e enfermeiros variou de 0,14 a 5,71 por 4.000 habitantes. Serviços com elevada densidade populacional possuem modelo operacional tradicional e déficit de profissionais. As regiões das llhas e do Extremo Sul, locais com elevada mortalidade infantil, apresentam, em maior área territorial, número adequado de médicos e enfermeiros. Observou-se elevada heterogeneidade na distribuição da mortalidade infantil (zero a 52,63 por 1.000 nascidos vivos) em 2014. Conclusão: Um terço da população de Porto Alegre é assistida pela saúde da família. Locais com elevada mortalidade infantil apresentaram número de médicos e enfermeiros próximo ao preconizado. Esse estudo é gerador de hipótese para futuras investigações epidemiológicas na área de atenção primária.

Palavras-chave: Atenção Primária à Saúde; Mortalidade Infantil; Saúde da População Urbana; Planejamento em Saúde; Mapeamento Geográfico
Janini Cristina Paiza

Marcio Bigolin ${ }^{b}$

Roger dos Santos Rosa ${ }^{a}$

Ronaldo Bordin ${ }^{\mathrm{a}}$

a Universidade Federal do Rio Grande do Sul (UFRGS). Porto Alegre, RS, Brasil. janinicpaiz@gmail.com; roger.rosa@bcb.gov.br; ronaldo.bordin@ufrgs.br

b Instituto Federal do Rio Grande do Sul (IFRS). Canoas, RS, Brasil. marcio.bigolin@canoas.ifrs.edu.br (Autor correspondente)
Como citar: Paiz JC, Bigolin M, Rosa RS, Bordin R. Mortalidade infantil e serviços de Atenção Primária à Saúde em Porto Alegre (RS), Brasil. Rev Bras Med Fam Comunidade. 2018;13(40):1-13. http://dx.doi.org/10.5712/rbmfc13(40)1579
Fonte de financiamento: declaram não haver.

Parecer CEP: não se aplica.

Conflito de interesses: declaram não haver. Procedência e revisão por pares: revisado por pares. Recebido em: 28/07/2017. Aprovado em: 06/06/2018. 


\begin{abstract}
Introduction: The Child Mortality Coefficient, an indicator of the social and health conditions of the population, has the capacity to guide the proposal of public policies, installation and forecasting of human resources in health. Territories with high infant mortality tend to be more vulnerable and require more structured primary care. Objective: To map infant mortality and describe the geographic distribution of primary care services and human resources (concentration and care "gaps") in Porto Alegre, in the years 2010 and 2014. Methods: Descriptive study, ecological design, employing secondary data available in health information systems. Data processing was performed by the Enyalius framework. Results: Of the 143 services, $65 \%$ have family health, with coverage of $36.5 \%$ of the population. The number of users adjoined per service ranges from 700 to 115,673 inhabitants; $35 \%$ of the services have less than 4,000 adjoined inhabitants. The distribution of doctors and nurses ranged from 0.14 to 5.71 per 4,000 inhabitants. Services with a high population density have a traditional operating model and a professional deficit. The regions of the Islands and of the Extreme South, places with high infant mortality, present, in a larger territorial area, an adequate number of doctors and nurses. There was a high heterogeneity in the distribution of infant mortality (zero to 52.63 per 1,000 live births) in 2014. Conclusion: One-third of the Porto Alegre population is assisted by family health. Locations with high infant mortality presented a number of doctors and nurses close to that recommended. This study generates hypotheses for future epidemiological investigations in the primary care area.
\end{abstract}

Keywords: Primary Health Care; Infant Mortality; Urban Health; Health Planning; Geographic Mapping

\title{
Resumen
}

Introducción: El Coeficiente de Mortalidad Infantil, indicador de las condiciones sociales y de salud de la población, tiene capacidad para orientar la proposición de políticas públicas, instalación y previsión de recursos humanos en salud. Los territorios con una elevada mortalidad infantil tienden a ser más vulnerables y a necesitar de una atención primaria bien estructurada. Objetivo: Mapear la mortalidad infantil y describir la distribución geográfica de los servicios y recursos humanos de atención primaria (concentración y "vacíos" asistenciales) en Porto Alegre, en los años 2010 y 2014. Métodos: Estudio descriptivo, delineamiento ecológico, con empleo de datos secundarios disponibles en sistemas de información en salud. El tratamiento de los datos ocurrió por el framework enyalius. Resultados: De los 143 servicios, $65 \%$ poseen salud de la familia, con cobertura del $36,5 \%$ de la población. El número de usuarios adscritos por servicio varía de 700 a 115.673 habitantes; El $35 \%$ de los servicios tienen menos de 4.000 habitantes adscritos. La distribución de médicos y enfermeros varió de 0,14 a 5,71 por 4.000 habitantes. Los servicios de alta densidad de población tienen un modelo operativo tradicional y un déficit de profesionales. Las regiones de las Islas y del extremo Sur, ubicadas con elevada mortalidad infantil, presentan, en mayor área territorial, número adecuado de médicos y enfermeros. Se observó una elevada heterogeneidad en la distribución de la mortalidad infantil (cero a 52,63 por 1.000 nacidos vivos) en 2014. Conclusión: Un tercio de la población de Porto Alegre es asistida por la salud de la familia. Los lugares con elevada mortalidad infantil presentaran un número de médicos y enfermeros próximo al previsto. Este estudio es generador de hipótesis para futuras investigaciones epidemiológicas en el área de atención primaria.

Palabras clave: Atención Primaria de Salud; Mortalidad Infantil; Salud Urbana; Planificación en Salud; Mapeo Geográfico

\section{Introdução}

AAtenção Primária à Saúde (APS) constitui uma importante forma de acesso do usuário às ações e serviços do Sistema Único de Saúde (SUS). A partir da Conferência Internacional sobre Cuidados Primários de Saúde, em Alma Ata (1978), a APS tem sido considerada como prioritária e capaz de resolver $80 \%$ das necessidades de saúde da população. ${ }^{1}$ Nesse contexto, Starfield ${ }^{2}$ definiu como atributos essenciais desse nível de atenção: (a) o primeiro contato do usuário, (b) a atenção integral, (c) a longitudinalidade e (d) a coordenação do cuidado.

O trabalho na APS, de acordo com o modelo organizacional de Estratégia de Saúde da Família (ESF), pressupõe o desenvolvimento de ações com alto grau de descentralização e capilaridade. ${ }^{3} A$ adscrição do território representa uma estratégia para vincular os usuários, a fim de produzir vínculos que possibilitem a atenção integral e longitudinal. ${ }^{3}$ Além disso, possibilita a identificação do grau de vulnerabilidade da população e garante que a alocação de recursos humanos para esses territórios sejam proporcionais às necessidades de atenção e redução das desigualdades existentes - equidade. . $^{3,4}$ 
Por atuarem em um território adscrito, as equipes de saúde da família possuem maior conhecimento dos determinantes e condicionantes dos processos de saúde-doença que envolvem a população da área, fator que facilita o acesso e o planejamento do cuidado, mas que, por si só, não é suficiente para reduzir as desigualdades na morbimortalidade de grupos sociais mais vulneráveis. ${ }^{5}$ A mortalidade infantil representa um agravo que poderia ser evitado na vigência de um sistema organizado e articulado que respondesse às necessidades de saúde da população. ${ }^{6}$ A relação entre redução da mortalidade infantil em famílias com condições socioeconômicas e de acesso ao serviço de saúde favoráveis já foi identificada por diversos estudos. ${ }^{7,8}$

Indicadores de saúde pública, como de mortalidade infantil, são instrumentos indispensáveis para definir as prioridades nas políticas públicas, planejar ações e serviços, monitorar a situação de saúde, bem como avaliar o desempenho do sistema de saúde. ${ }^{9}$ Assim, devem ser mapeados, a fim de identificar o local de ocorrência, e utilizados como informação para planejar as ações em saúde pública.

O georreferenciamento (análise espacial), utilizado inicialmente em $1854,{ }^{10}$ é um instrumento capaz de identificar a distribuição geográfica de eventos relacionados à saúde coletiva e de facilitar o planejamento e intervenção sobre esses agravos. Sua utilização na área da saúde é descrita como auxílio no processo de trabalho das equipes de saúde (vigilância de doenças e mapeamento do perfil do território) e na localização de eventos ambientais e condições sociais e seus impactos na saúde pública. ${ }^{11-13}$

Nesse contexto, o presente estudo objetiva mapear em territórios geográficos do município de Porto Alegre-RS, Brasil, a mortalidade infantil e a capacidade instalada de serviços de APS e de recursos humanos desse nível de atenção, a fim de identificar "vazios" assistenciais e locais com concentração de recursos humanos. Esse estudo busca associar os achados de concentração e escassez de capacidade operativa dos serviços de saúde com locais de elevada incidência de mortalidade infantil, a fim de evidenciar aspectos inerentes à equidade na distribuição dos serviços de saúde.

\section{Métodos}

Trata-se de um estudo descritivo de delineamento ecológico, com emprego de dados secundários, disponíveis em bases de acesso público: Cadastro Nacional de Estabelecimentos de Saúde (CNES), Instituto de Pesquisa Econômica Aplicada (IPEA), Programa das Nações Unidas para o Desenvolvimento (PNUD) - Atlas do Desenvolvimento Humano no Brasil e do Observatório da Cidade de Porto Alegre. O período de coleta de dados ocorreu de agosto de 2015 a maio de 2016.

Porto Alegre é a capital do estado Rio Grande do Sul. De acordo com Censo do Instituto Brasileiro de Geografia e Estatística (IBGE), a população do município era de 1.409.351 habitantes em 2010, com um Índice de Desenvolvimento Humano de 0,805. ${ }^{14}$

Os dados referentes à capacidade instalada de serviços de APS do município (Unidades Básicas de Saúde - UBS e Unidades de Saúde da Família - USF), bem como a delimitação dos territórios e da população adscrita por UBS/USF, estão disponíveis no Observatório de Porto Alegre, página Web atualizada periódicamente pela prefeitura municipal. 
Os dados referentes à mortalidade infantil em 2010 estão disponíveis no Atlas do Desenvolvimento Humano no Brasil. Esse sistema contém indicadores de demografia, educação, renda, trabalho, habitação e vulnerabilidade, com dados extraídos dos Censos Demográficos de 1991, 2000 e 2010. Foram utilizados os Coeficientes de Mortalidade Infantil (CMI) das 335 Unidades de Desenvolvimento Humano (UDHs) de Porto Alegre. As UDHs são áreas que podem ser parte ou um bairro completo, formadas a partir da agregação dos setores censitários do IBGE. Os limites são definidos pela homogeneidade socioeconômica.

Também foi utilizado o número de óbitos infantis (menores de um ano) e o número de nascidos vivos na cidade em 2014, para cálculo do CMI, disponíveis no Observatório do de Porto Alegre. ${ }^{15}$ Os dados referentes à distribuição dos recursos humanos que compõem as UBS/USF do município foram obtidos por meio do CNES (base setembro/2015), considerando-se as seguintes categorias profissionais: médicos, enfermeiros, técnicos/auxiliares de enfermagem e Agentes Comunitários de Saúde (ACS). Ou seja, as categorias profissionais tipicamente previstas em uma equipe de ESF. ${ }^{3}$

Para identificar a adequação dos recursos humanos nos diferentes espaços geográficos, foi utilizado o número de equipes completas para atender determinada densidade populacional. $O$ indicador de cobertura foi o previsto pela normatização vigente - cada equipe deve ser responsável por, no máximo, 4.000 pessoas. ${ }^{3}$ Para cálculo de adequação do número de ACS, utilizou-se a recomendação de 750 usuários vinculados a esse profissional. ${ }^{3}$

Os dados foram provenientes de diferentes fontes, assim o tratamento foi realizado de diversas maneiras. Na primeira filtragem, os dados foram inseridos em uma planilha eletrônica (Microsoft ${ }^{\circledR}$ Excel 2010) e, posteriormente, armazenados em um banco de dados relacional utilizando o Sistema Gerenciador de Bancos de Dados (SGBD) de uso comum PostgreSQL. Já os dados do CNES, por exigirem diversas consultas, foram catalogados de maneira automática. Nessa etapa, foi necessário desenvolver uma ferramenta que utilizou a linguagem Hypertext Preprocessor (PHP) e o framework Enyalius, que tem como base um conjunto de bibliotecas para a geração de gráficos, relatórios e mapas, entre outros. Com a ferramenta desenvolvida, foram realizados os cálculos e gerados os mapas apresentados nesse trabalho.

Para a obtenção das coordenadas geográficas das UBS/ESF foram utilizados os endereços das mesmas, os quais foram submetidos ao WebService do Google Maps. Esse WebService retorna as informações das coordenadas geográficas a partir do endereço. As coordenadas foram armazenadas no banco de dados (SGBD), sendo os mapas gerados por meio da ferramenta com o auxílio do Enyalius.

Os dados demográficos específicos de cada UBS/USF, obtidos por meio da consulta em informações de saúde do Observatório de Porto Alegre, foram agrupados e relacionados com as respectivas localizações geográficas de cada UBS/USF. O mesmo ocorreu com os recursos humanos por serviço de APS.

Este estudo não necessitou encaminhamento para apreciação por Comitê de Ética em Pesquisa (CEP), uma vez que utilizou dados secundários provenientes de bases de acesso público para o seu desenvolvimento, não apresentando riscos de qualquer espécie aos seres humanos. ${ }^{16}$ 


\section{Resultados}

Na Figura 1, encontra-se a distribuição da população e dos serviços de atenção primária.

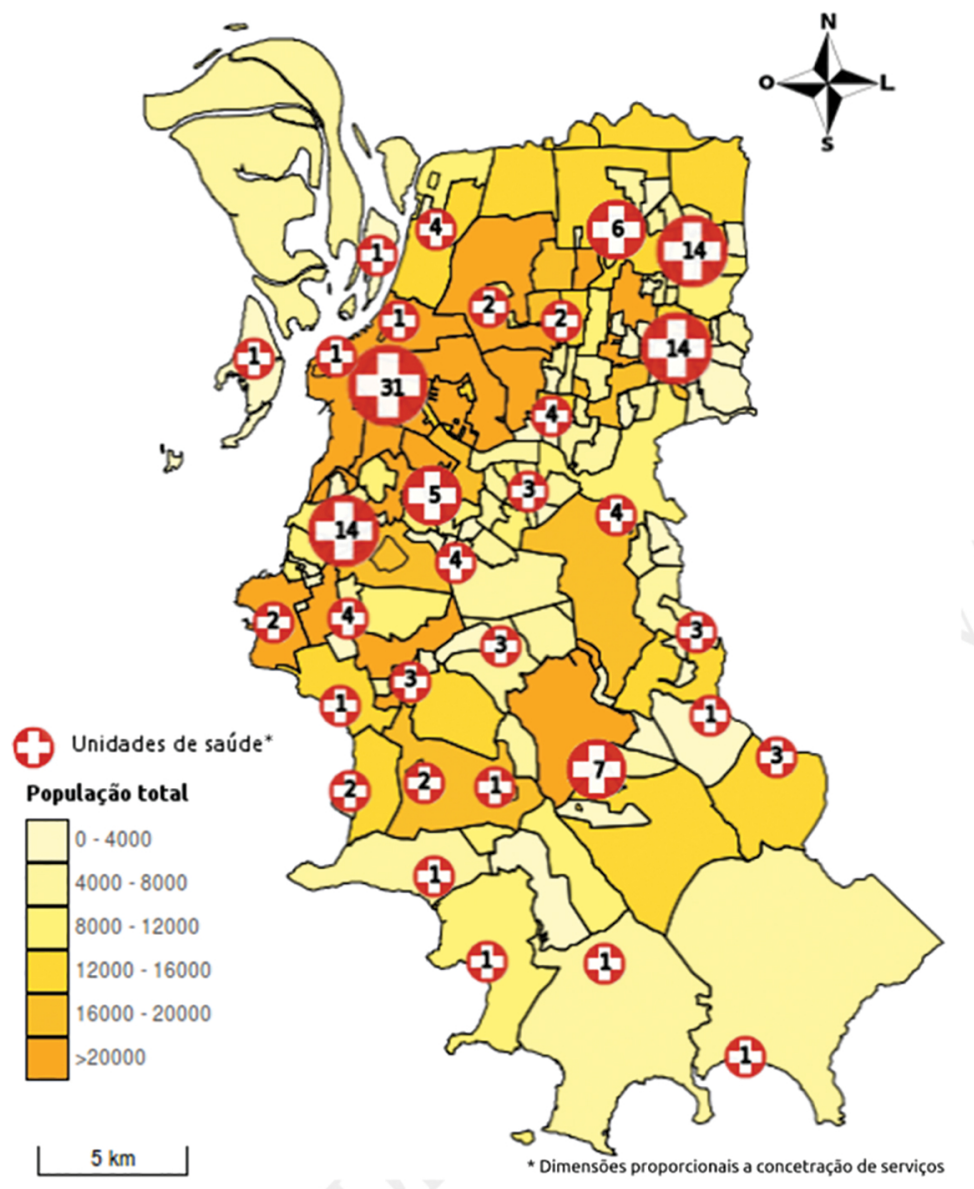

Figura 1. Distribuição da população e dos serviços de APS no município de Porto Alegre, 2014. Fonte: Elaborado por meio de dados do Observatório de Porto Alegre, 2014

A rede de APS do município é composta por 143 serviços, dos quais 50 (35\%) possuem população adscrita igual ou inferior a 4 mil habitantes, $43(30 \%)$ entre 4 e 8 mil habitantes, $16(11,2 \%)$ entre 8 e 12 mil habitantes, 14 (9,8\%) entre 12 e 16 mil habitantes, 8 (5,6\%) entre 16 e 20 mil habitantes e 12 (8,4\%) superior a 20 mil habitantes.

Os estabelecimentos que possuem equipes de saúde da família e equipes tradicionais foram contabilizados com um único serviço para cálculo de porcentagem, uma vez que suas equipes estavam cadastradas em um único CNES, mas estão apresentados no mapa de acordo com a população adscrita por ESF e por UBS tradicional. Casos em que os serviços possuem, além de CNES, divisão territorial e especificação dos profissionais para cada modelo de atenção (ESF ou tradicional), foram considerados serviços diferentes. 
A Figura 2 evidencia os territórios em que o processo de trabalho é organizado por meio das equipes de saúde da família e os territórios em que não há cobertura de ESF, ou seja, são UBS tradicionais com responsabilidade sobre o território. Também delimita os territórios em que a capacidade de recursos humanos da ESF está de acordo com a PNAB para assistir a área adscrita. ${ }^{3}$

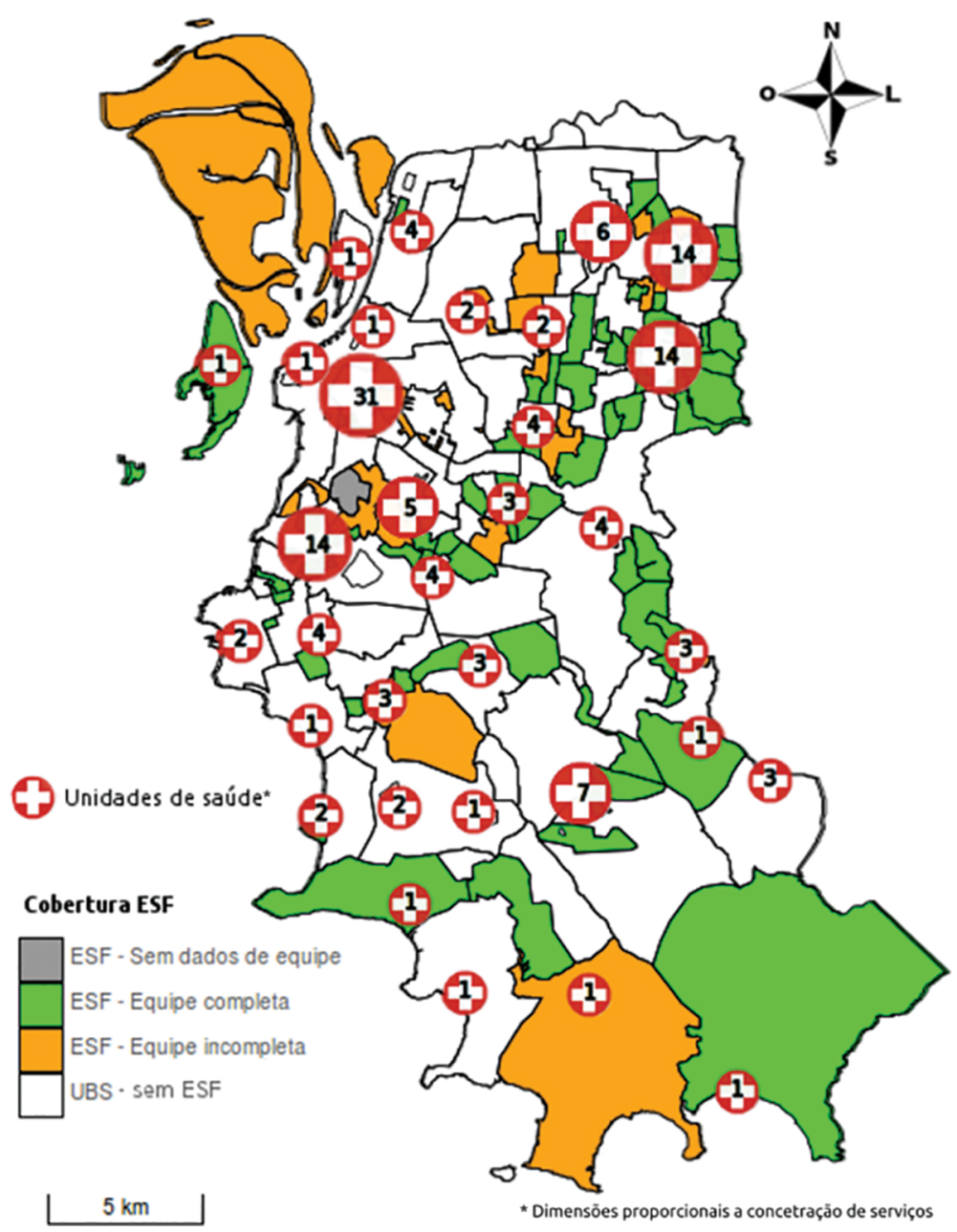

Figura 2. Cobertura de ESF em Porto Alegre, 2015. Fonte: Elaborado por meio de dados obtidos no CNES, 2015.

De acordo com o Plano Municipal de Saúde, 46\% da população de Porto Alegre residia em área coberta por ESF em 2014. ${ }^{17}$ Entretanto, na Figura 2, obteve-se resultado distinto, com apenas $36,5 \%$ da população assistida por ESF. Em dados absolutos, de 1.416.419 habitantes, apenas 516.998 teriam cobertura de ESF.

Dentre os 143 serviços de APS do município, 46 (32,2\%) são UBS tradicionais, 93 (65\%) são UBS com ESF e quatro têm parte da população atendida por ESF e parte por UBS tradicional. Dentre as 97 ESF, $18(18,6 \%)$ possuem equipes incompletas. Considerado os dados provenientes do CNES de 2015 em relação aos recursos humanos das ESF, percebe-se um número insuficiente de ACS. ${ }^{3}$ 
A Figura 3 apresenta a concentração de profissionais de nível superior, componentes da equipe mínima de ESF prevista na PNAB ${ }^{3}$ - médicos generalistas ou especialistas em saúde de família e/ou comunidade e enfermeiro - com carga horária mínima de 30h ou com equivalência mínima de 30h realizada por dois ou mais profissionais.
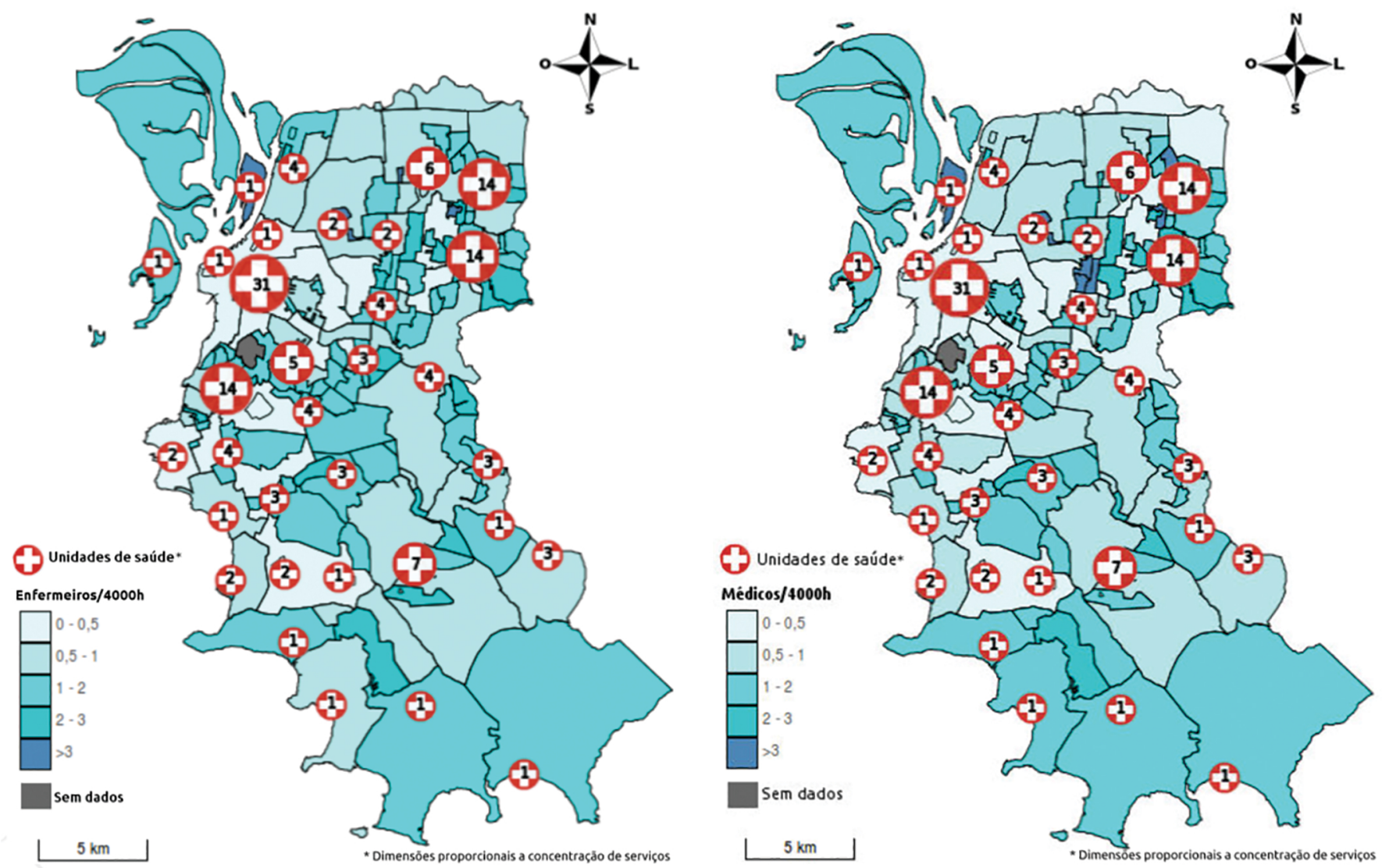

Figura 3. Concentração de médicos e enfermeiros no município de Porto Alegre, 2015. Fonte: Elaborado por meio de dados obtidos no CNES, 2015.

A distribuição dos médicos variou de 0,14 a 5,71/4 mil habitantes, enquanto de enfermeiros variou de 0,20 a 5,71/4 mil habitantes, evidenciando heterogeneidade na distribuição desses profissionais (Figura 3). Os dados de carga horária do profissional médico no CNES revelam uma fragmentação do processo de trabalho e da proposta de composição das ESF. Os serviços sem implantação de ESF apresentam a maioria das particularidades, tais como especialistas de diversas áreas, profissionais com carga horária semanal variada (de 2, 5, 7, 8, 10, 12, 18 horas), mesmo profissional com vínculos de 40 horas em dois serviços diferentes e no mesmo serviço.

A Figura 4 apresenta um dos indicadores de maior validade no planejamento e implementação de políticas públicas pela capacidade de refletir as condições sociais e de saúde da população. Por meio dessa Figura, é possível visualizar comparativamente o CMI nos diferentes territórios, nos anos de 2010 e 2014. 

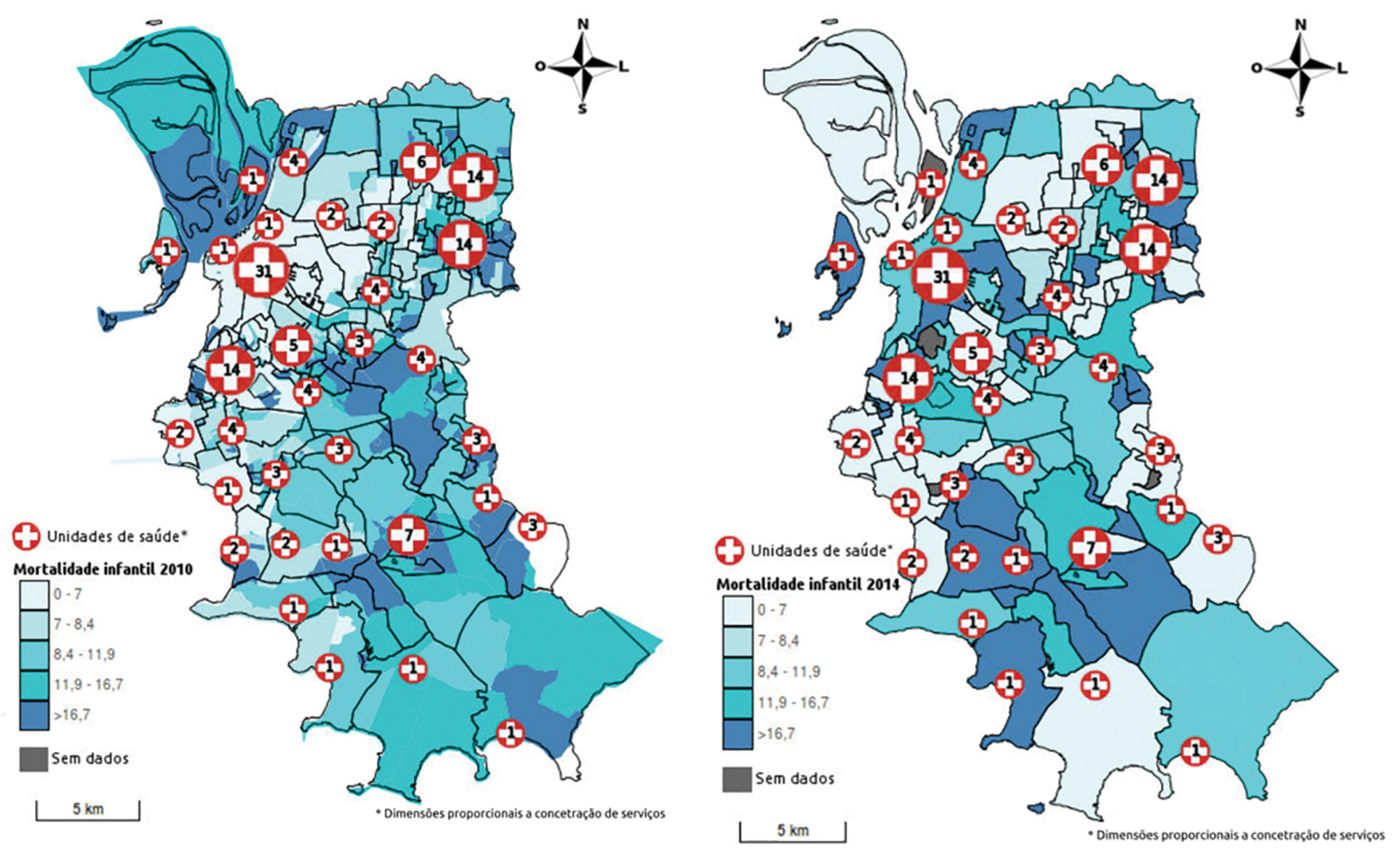

Figura 4. Coeficiente de Mortalidade Infantil (CMI) em Porto Alegre em 2010 e 2014. Fonte: Mortalidade em 2010 dados do Atlas do Desenvolvimento Humano no Brasil, 2010; Mortalidade infantil 2014 dados do Observatório da cidade de Porto Alegre, 2014.

Em 2010, a região Central apresentou o menor CMI (variação de 0 a 7 óbitos/mil nascidos vivos) enquanto as regiões Sul e das Ilhas apresentaram as piores taxas (CMI igual ou superior à média nacional - 16,7 óbitos/mil nascidos vivos). Em 2014, observa-se maior heterogeneidade nos territórios. A região Sul permanece apresentando áreas de elevado $\mathrm{CMI}$, bem como parte da região das Ilhas, mas, diferentemente de 2010, a região Central passa a se configurar como território de maior coeficiente de mortalidade infantil.

A elevada heterogeneidade na distribuição do CMI é um dos aspectos principais a serem observados. O coeficiente variou de 0 a 52,63 óbitos/1.000 nascidos vivos em 2014. Os maiores CMI observados nas áreas das Unidades de Saúde foram: 52,63, 50,0, 43,47 e 41,66 óbitos/mil nascidos vivos.

\section{Discussão}

\section{Resumo dos achados}

O municipio possui 141 estabelecimentos físicos de APS, organizados em 147 UBS tradicionais/USF. Há seis estabelecimentos que compartilham o mesmo espaço físico para atendimento da população, com modelo de atenção tradicional e ESF. Há concentração de população e de serviços de APS na região central (Centro Histórico). Ao mesmo tempo, é possível identificar que um extenso território, abrigando $23,8 \%$ dos estabelecimentos de APS, possui mais de 12 mil pessoas adscritas. 
Os serviços de APS que concentram maior número de pessoas sob sua responsabilidade são UBS tradicionais, cujas três maiores possuem 115.673, 101.917 e 58.252 habitantes adscritos. Caracterizam-se por possuir equipes de atenção básica mistas, com profissionais especializados, tais como médicos pediatras e ginecologistas.

Serviços de APS com elevado número de usuários adscritos e com presença de profissionais especialistas integrando as equipes podem sofrer uma fragmentação do trabalho de vigilância do território e comprometer alguns dos princípios da APS. A atenção longitudinal, por exemplo, pode estar comprometida, à medida que a equipe de ESF transfere a responsabilidade pelo cuidado da criança em puericultura para o pediatra, ou, ainda, da gestante de baixo risco para o ginecologista.

A presença do especialista no serviço pode melhorar a qualidade da atenção quando dividir responsabilidades e práticas com as equipes de ESF - modalidade de organização conhecida como matriciamento. Entretanto, a elevada demanda do serviço e a comodidade dos profissionais podem levar à criação de uma agenda segmentada e desvinculada do cuidado previsto na APS.

No outro extremo, 50 serviços possuem população adscrita inferior a 4 mil habitantes e eixo organizador do processo de trabalho a ESF. As 3 USF com menor número de usuários vinculados possuem 700 habitantes, 1520 habitantes e 1569 habitantes adscritos. A baixa densidade populacional permite maior reconhecimento do território como cenário para atuação frente às potencialidades e vulnerabilidades a ele inerentes.

Em relação aos recursos humanos, percebe-se que o município possui dimensionamento inadequado, com áreas em que há número insuficiente de profissionais para atender à densidade populacional, os chamados "vazios assistenciais", bem como territórios com concentração de profissionais acima do parâmetro indicado pela PNAB.

O cenário atual, que envolve a distribuição heterogênea de recursos humanos, consiste em uma problemática multifatorial. Áreas periféricas, distantes da região central e com elevado índice de violência, possuem uma tendência a apresentar menor número de profissionais vinculados. Entretanto, na Figura 3 é nítida a observação da região central como território de baixa densidade profissional e elevada população residente. Por apresentar um poder aquisitivo elevado, com usuários vinculados a seguradoras/planos de saúde, fatores que não foram avaliados nesse estudo, apresenta comportamento diferenciado quanto ao emprego do serviço público de saúde. Observa-se pelos mapas que locais de elevada mortalidade infantil, como região das Ilhas e o Extremo Sul, possuem um número relativamente adequado de médicos e enfermeiros.

O coeficiente de mortalidade infantil, como indicador das condições de vida e saúde da população, permite identificar a vulnerabilidade que afeta de maneira heterogênea o município. Percebe-se que há elevada heterogeneidade na distribuição do CMI, que varia de 0 a 52,63 óbitos/1.000 nascidos vivos em 2014. Os maiores CMI observados nas áreas das Unidades de Saúde foram: 52,63, 50,0, 43,47 e 41,66 óbitos/mil nascidos vivos. E, desta forma, este indicador, segundo o apresentado pelos mapas (Figuras 3 e 4), não foi o principal aspecto a ser considerado na implantação de serviços e recursos humanos à saúde no município. 


\section{Limitações}

Entre as limitações desse estudo estão a utilização de dados secundários (delineamento ecológico, com baixo poder de inferência causal) e de diferentes sistemas de informações para comparação dos dados referentes à mortalidade infantil nos anos de 2010 e 2014 e associação da mortalidade infantil restrita à capacidade instalada (física e de recursos humanos) da APS. É consenso que a mortalidade infantil é resultado de múltiplos fatores (socioeconômicos, culturais, acesso a recursos e benefícios públicos, escolaridade materna, entre outros), entretanto, esse estudo restringiu-se a associar a mortalidade infantil com a capacidade instalada de serviços de APS.

\section{Comparação com a literatura}

A implantação da ESF nos territórios brasileiros tem demonstrado diversos graus de adequação aos cenários de atenção. Em centros urbanos, a evolução da cobertura ocorre de maneira incipiente. ${ }^{18} \mathrm{~A}$ complexidade na organização dos serviços de saúde em grandes centros traz obstáculos no que se refere ao acesso equânime e à garantia da atenção integral. Essa complexidade diz respeito ao elevado grau de exclusão do acesso aos serviços de saúde (insuficiente capacidade física e de recursos humanos), aos agravos de saúde característicos das grandes cidades e metrópoles e de uma oferta consolidada em redes assistenciais desarticuladas e mal distribuídas, em parte devido à fragilidade da APS em exercer o papel de coordenadora da rede..$^{19-21}$

O dimensionamento adequado de profissionais de acordo com critérios populacionais e com a vulnerabilidade do território é fator fundamental para fomentar processos de construção de vínculos longitudinais e para possibilitar a atenção integral à saúde da população adscrita. Permite também melhor acesso e qualidade da atenção e impacta na melhoria de indicadores diretos e indiretos de saúde e de qualidade da atenção. A escassez de recursos humanos, materiais, infraestrutura e de comunicação/informação são citados em estudos que identificam as percepções dos trabalhadores da ESF. ${ }^{22}$

A gama de ações oferecidas às crianças em seus primeiros anos de vida pelos serviços de APS, em especial quando elas residem com suas famílias em territórios com cobertura de ESF, leva à redução da mortalidade infantil. ${ }^{23}$ Os achados neste estudo corroboram pesquisa realizada na região Sudeste do Brasil que relacionou a capacidade instalada de serviços de APS com critérios de vulnerabilidade. Naquela investigação, foi constatada elevada heterogeneidade e cumprimento não satisfatório da distribuição equânime de serviços de APS. ${ }^{24}$

A mortalidade infantil apresentou redução importante em todas as regiões brasileiras (decréscimo anual: $6 \%$ Nordeste; $4,1 \%$ Norte e Sudeste; 3,9\% Sul; e 3,2\% Centro-Oeste), fator atribuído à melhoria das condições de vida da população brasileira - programas sociais de redistribuição de renda e maior acesso e qualidade da assistência no pré-natal, parto e puerpério. ${ }^{25}$ Em Porto Alegre, o CMI passou de 11,6 em 2010 para 9,5 em 2014, uma redução de $18 \%$ no período.

Mesmo com avanços, enfatiza-se que os desafios atuais para a redução da mortalidade infantil encontram-se na qualificação da atenção ao pré-natal, na redução da gravidez na adolescência, na prevenção da prematuridade e melhoria da qualidade da atenção hospitalar ao recém-nascido prematuro. ${ }^{26}$ 


\section{Fortalezas e implicações para a prática profissional}

A principal contribuição deste estudo decorre de sua aplicabilidade na área da gestão em saúde, especificamente na subárea de epidemiologia dos serviços de saúde, ao fornecer subsídios para a alocação ou adequação da capacidade instalada e dos recursos humanos em saúde de acordo com um indicador (CMI) de vulnerabilidade e equidade. Por permitir a apresentação de dados em forma gráfica de um indicador sensível à condição social e de saúde da população e demostrar a realidade atual das grandes cidades (heterogeneidade na distribuição da população, elevada densidade populacional em determinadas regiões e vazios assistenciais), configura-se em uma ferramenta para diagnóstico e planejamento no que se refere à saúde em grandes centros.

Esse estudo também identificou fragilidades no sistema de cadastro de profissionais. Cabe destacar que o cadastro adequado dos profissionais que compõem a ESF, para os serviços que aderiram à proposta, é critério indispensável para o repasse de verbas referentes ao PAB variável da Atenção Básica. As fragilidades encontradas no acesso ao CNES refletem a necessidade de rever as atualizações de profissionais que não mais fazem parte do serviço, incluir novos trabalhadores, excluir cadastros repetidos e revisar as cargas horárias digitalizadas.

\section{Conclusões}

Este estudo identificou que:

a) $36,5 \%$ da população de Porto Alegre é assistida pela saúde de família (516.998 habitantes); heterogeneidade na distribuição de médicos e enfermeiros para a atenção básica, com déficit desses profissionais em regiões de elevada densidade populacional, ainda que com número próximo do adequado em regiões de maior mortalidade infantil, tais como regiões das llhas e do Extremo Sul.

As equipes de atenção básica tradicionais atuam como uma estratégia "paliativa" para dar acesso a um conjunto básico e fragmentado de ações e cuidados em saúde e apresentam limitações na atuação no território de maneira organizada e preventiva. $O$ déficit de profissionais na região de elevada densidade populacional diz respeito a um número ainda insuficiente de serviços para atender as demandas dos aglomerados populacionais. O município possui $23,8 \%$ dos estabelecimentos de APS com mais de 12 mil pessoas adscritas.

b) Comparando-se o final ao início do quinquênio 2010-2014 (Figura 4), parece haver maior heterogeneidade nos territórios quanto ao coeficiente de mortalidade infantil (variou de zero a 52,6 óbitos por mil nascidos vivos).

Cabe enfatizar que este trabalho consiste em uma avaliação ecológica do cenário em estudo. Nesse sentido, essa conclusão deve ser considerada com certa limitação. A observação da Figura 4 mostra um cenário com heterogeneidade de mortalidade infantil nos diferentes anos avaliados, entretanto, é necessária a realização de estudos de maior consistência metodológica para confirmação da hipótese levantada nessa pesquisa. 
c) Os serviços de APS que concentram maior número de pessoas sob sua responsabilidade (acima de 50 mil habitantes) caracterizam-se por possuir equipes de atenção básica mistas, com profissionais especializados, como médicos pediatras e ginecologistas.

Serviços de atenção primária com elevado contingente populacional vinculado (acima de 50 mil habitantes) consistem em cenários precarizados, com baixa capacidade de atuação no território e desempenho de um trabalho fragmentado. Urge a necessidade de o poder público olhar para esses cenários e atuar sobre a insuficiência de capacidade física e de pessoal para produção de cuidado em atenção primária.

\section{Referências}

1. World Health Organization (WHO). Primary Health Care. Geneva: World Health Organization; 1978.

2. Starfield B. Atenção Primária. Equilíbrio Entre Necessidade de Saúde, Serviços e Tecnologia. Brasília: UNESCO, Ministério da Saúde; 2002. 726 p [acesso 2018 Mar 20]. Available from: https://www.nescon.medicina.ufmg.br/biblioteca/imagem/0253.pdf

3. Brasil. Ministério da Saúde. Política Nacional de Atenção Básica (PNAB). Brasília: Ministério da Saúde; 2012 [acesso 2018 Mar 20 ]. Available from: http://189.28.128.100/dab/docs/publicacoes/geral/pnab.pdf

4. Vianna SM, Nunes A, Santos JRS, Barata RB. Medindo as desigualdades em saúde no Brasil: uma proposta de monitoramento. 1a ed. Brasília: OPAS/OMS; 2001.

5. Pelegrini MLM, Castro JD, Drachler ML. Eqüidade na alocação de recursos para a saúde: a experiência do Estado do Rio Grande do Sul, Brasil. Ciên Saúde Coletiva 2005;10(2):275-86. http://dx.doi.org/10.1590/S1413-81232005000200002

6. Maia LTDS, Souza WV, Mendes ADCG. Diferenciais nos fatores de risco para a mortalidade infantil em cinco cidades brasileiras: um estudo de caso-controle com base no SIM e no SINASC. Cad Saúde Pública. 2012;28(11):2163-76. http://dx.doi.org/10.1590/S0102311X2012001100016

7. Brasil. Ministério da Saúde. Atenção à Saúde do Recém-Nascido: Guia para os profissionais de saúde. Departamento de ações programáticas e estratégicas. Brasilía: Ministério da Saúde; 2011.

8. Lourenço EDC, Guerra LM, Tuon RA, Vidal Silva SMC, Ambrosano GMB, Corrente JE, et al. Variáveis de impacto na queda da mortalidade infantil no Estado de São Paulo, Brasil, no período de 1998 a 2008. Ciên Saúde Coletiva. 2014;19(7):2055-62. http://dx.doi. org/10.1590/1413-81232014197.18822013

9. Frias PG, Szwarcwald CL, Lira PIC. Estimação da mortalidade infantil no contexto de descentralização do Sistema Único de Saúde (SUS). Rev Bras Saúde Matern Infant. 2011;11(4):463-70. http://dx.doi.org/10.1590/S1519-38292011000400013

10. Câmara G, Monteiro AMV, Druck S, Carvalho MS. Análise Espacial e Groprocessamento. In: Druck S, Carvalho MS, Câmara G, Monteiro AVM, eds. Análise Espacial de Dados Geográficos. Brasília: Embrapa; 2004.

11. Magalhães MAFM, Matos VP, Medronho RA. Avaliação do dado sobre endereço no Sistema de Informação de Agravos de Notificação utilizando georreferenciamento em nível local de casos de tuberculose por dois métodos no município do Rio de Janeiro. Cad Saúde Coletiva. 2014;22(2):192-9. http://dx.doi.org/10.1590/1414-462X201400020013

12. Xavier DR, Barcellos C, Barros HS, Magalhães MAFM, Matos VP, Pedroso MM. Organização, disponibilização e possibilidades de análise de dados sobre desastres de origem climática e seus impactos sobre a saúde no Brasil. Ciênc Saúde Coletiva. 2014;19(9):3657-68. http://dx.doi.org/10.1590/1413-81232014199.00992014

13. Silva JV, Machado FCA, Ferreira MAF. As desigualdades sociais e a saúde bucal nas capitais brasileiras. Ciênc Saúde Coletiva. 2015;20(8):2539-48. http://dx.doi.org/10.1590/1413-81232015208.12052014

14. Brasil. Instituto Brasileiro de Geografia e Estatística (IBGE). Rio Grande do Sul: IBGE; 2016.

15. Brasília. Indicadores básicos para a saúde no Brasil: Conceitos e aplicações. 2a ed. [Internet]. Brasília: Organização Pan-Americana da Saúde; 2008. 350 p [acesso 2018 Mar 20]. Available from: http://tabnet.datasus.gov.br/tabdata/livroidb/2ed/indicadores.pdf 
16. Brasil. Conselho Nacional de Saúde. Resolução No 466, de 12 de dezembro de 2012. Aprova as diretrizes e normas regulamentadoras de pesquisas envolvendo seres humanos. Diário Oficial da União. Brasília; 2012.

17. Prefeitura Municipal de Porto Alegre. Secretaria Municipal de Saúde. Plano Municipal de Saúde 2014 - 2017. Porto Alegre; 2014.

18. Magnago C, Pierantoni CR. Dificuldades e estratégias de enfrentamento referentes à gestão do trabalho na Estratégia Saúde da Família, na perspectiva dos gestores locais: a experiência dos municípios do Rio de Janeiro (RJ) e Duque de Caxias (RJ). Saúde Debate. 2015;39(104):9-17. http://dx.doi.org/10.1590/0103-110420151040194

19. Escorel S, Giovanella L, Mendonça MHM, Senna MCM. O Programa de Saúde da Família e a construção de um novo modelo para a atenção básica no Brasil. Rev Panam Salud Publica. 2007;21(2):164-76. http://dx.doi.org/10.1590/S1020-49892007000200011

20. Santos MA, Senna MCM. Atenção Primária à Saúde: centralidade na agenda pública brasileira e desafios contemporâneos. Rev Uniabeu. 2015;8(19):337-53 [acesso 2018 Mar 20]. Available from: http://revista.uniabeu.edu.br/index.php/RU/article/view/1996/pdf_250

21. Conill EM. A historical and conceptual model for Primary Health Care: challenges for the organization of primary care and the Family Health Strategy in large Brazilian cities. Cad Saúde Pública. 2008;24 Suppl 1:S7-16. http://dx.doi.org/10.1590/S0102-311X2008001300002

22. Silva LMS, Fernandes MC, Mendes EP, Evangelista NC, Torres RAM. Trabalho interdisciplinar na estratégia saúde da família: enfoque nas ações de cuidado e gerência. Rev Enferm UERJ. 2012;20(n.esp.2):784-8 [acesso 2018 Mar 20]. Available from: http://www.e-publicacoes. uerj.br/index.php/enfermagemuerj/article/view/6024/4329

23. Stábile AP, Braz JC, Furtado MCC, Mello DF. Indicadores de saúde infantil na estratégia saúde da família no Brasil: revisão integrativa da literatura. Rev Ciênc Méd. 2013;22(1):31-41 [acesso 2018 Mar 20]. Available from: https://seer.sis.puc-campinas.edu.br/seer/index. php/cienciasmedicas/article/view/1999

24. Braga GB, Aurélio M, Marques F, Braga BB. Análise da distribuição espacial das estruturas voltadas à Atenção Primária à Saùde do Sudeste Brasileiro. Espaç Saúde. 2015;3(16):14-26.

25. Frias PG, Szwarcwald CL, Souza Junior PRB, Almeida WS, Lira PIC. Correcting vital information: Estimating infant mortality, Brazil, 2000-2009. Rev Saude Publica. 2013;47(6):1048-58. http://dx.doi.org/10.1590/S0034-8910.2013047004839

26. Pizzo LGP, Andrade SM, Silva AMR, Melchior R, González AD. Mortalidade infantil na percepção de gestores e profissionais de saúde: determinantes do seu declínio e desafios atuais em município do sul do Brasil. Saude Soc. 2014;23(3):908-18. http://dx.doi.org/10.1590/ S0104-12902014000300014 